\title{
Collision-induced conformational changes in glycine
}

\author{
Thomas F. Miller III ${ }^{\text {a) }}$ and David C. Clary \\ Department of Physical and Theoretical Chemistry, Oxford University, South Parks Road, Oxford OX1 3QZ, \\ United Kingdom
}

Anthony J. H. M. Meijer ${ }^{\text {b) }}$

Department of Chemistry, University of Sheffield, Sheffield S3 7HF, United Kingdom

(Received 17 March 2005; accepted 15 April 2005; published online 6 July 2005)

\begin{abstract}
We present quantum dynamical calculations on the conformational changes of glycine in collisions with the $\mathrm{He}, \mathrm{Ne}$, and Ar rare-gas atoms. For two conformer interconversion processes (III $\rightarrow \mathrm{I}$ and $\mathrm{IV} \rightarrow \mathrm{I}$ ), we find that the probability of interconversion is dependent on several factors, including the energy of the collision, the angle at which the colliding atom approaches the glycine molecule, and the strength of the glycine-atom interaction. Furthermore, we show that attractive interactions between the colliding atom and the glycine molecule catalyze conformer interconversion at low collision energies. In previous infrared spectroscopy studies of glycine trapped in rare-gas matrices and helium clusters, conformer III has been consistently observed, but conformer IV has yet to be conclusively detected. Because of the calculated thermodynamic stability of conformer IV, its elusiveness has been attributed to the IV $\rightarrow$ I conformer interconversion process. However, our calculations present little indication that IV $\rightarrow$ I interconversion occurs more readily than III $\rightarrow$ I interconversion. Although we cannot determine whether conformer IV interconverts during experimental Ne- and Ar-matrix depositions, our evidence suggests that the conformer should be present in helium droplets. Anharmonic vibrational frequency calculations illustrate that previous efforts to detect conformer IV may have been hindered by the overlap of its IR-absorption bands with those of other conformers. We propose that the redshifted symmetric $-\mathrm{CH}_{2}$ stretch of conformer IV provides a means for its conclusive experimental detection.
\end{abstract}

(C) 2005 American Institute of Physics. [DOI: 10.1063/1.1927527]

\section{INTRODUCTION}

Glycine is the simplest amino acid. ${ }^{1,2}$ Because of its biological relevance and small size, it is a valuable prototype for the theoretical and experimental studies of molecular conformation. $^{2-28}$ Moreover, the recent detection of glycine in the interstellar medium has intensified interest in its gasphase dynamics. ${ }^{29}$ Extensive electronic structure theory studies predict glycine to have numerous stable conformers arising from internal rotation about its three torsional modes, ${ }^{11-13}$ but only three of these conformers have been conclusively observed in experiment. ${ }^{18-23}$ In the current paper, we present a theoretical study of collision-induced conformational changes in glycine and consider evidence for the population of a fourth conformer.

Figure 1(a) presents the experimentally observed conformers of glycine and their thermal populations at $438 \mathrm{~K}$, as determined from the integrated $\nu(\mathrm{C}=\mathrm{O})$ bands of the infrared vibrational spectrum taken in a low-temperature neon matrix. $^{22}$ We recently used the quantum torsional pathintegral Monte Carlo (TPIMC) method ${ }^{30,31}$ to accurately reproduce the observed populations of conformers II and III. ${ }^{26}$ However, in addition to the three conformers shown in

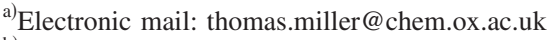

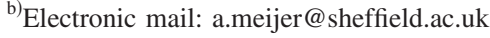

Fig. 1(a), our calculations predicted a fourth conformer of glycine [Fig. 1(b)] to be substantially populated. We found that agreement for the population of conformer I was reached only after assuming that the total population of conformer IV interconverts to conformer I prior to spectroscopic measurement. These results are summarized in Table I.

Conformer interconversion is a complication often encountered in the gas-phase spectroscopy of biological molecules. ${ }^{17,32-35}$ It arises from the need to both (a) heat the sample of molecules to obtain a gas-phase concentration that is sufficient for spectroscopic measurement and (b) cool the molecules to obtain a clear spectrum. This is illustrated for a typical IR or microwave spectroscopy experiment in Fig. 2. In the upper panel, an oven is used to vaporize a sample of the molecules of interest in the presence of an inert gas,

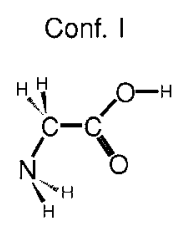

(a)

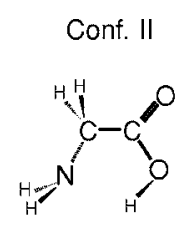

$11 \%$
Conf. III

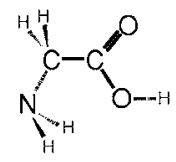

$17 \%$

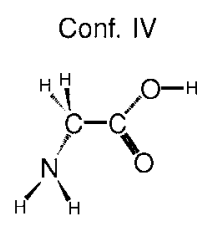

(b)
Not observed
FIG. 1. The conformers of glycine. (a) Conformer populations at $438 \mathrm{~K}$ determined from neon-matrix infrared spectroscopy (see Ref. 22). (b) Conformer IV not observed experimentally. 
TABLE I. Comparison of the experimental ${ }^{\mathrm{a}}$ and calculated $^{\mathrm{b}}$ conformer populations $(\%)$ of glycine ${ }^{\mathrm{c}}$.

\begin{tabular}{|c|c|c|c|c|c|c|}
\hline Conf. & Expt. & $\begin{array}{c}358 \mathrm{~K} \\
\text { Calc. } \\
\text { without } \mathrm{IC}^{\mathrm{d}}\end{array}$ & $\begin{array}{c}\text { Calc. } \\
\text { with } \mathrm{IC}^{\mathrm{a}}\end{array}$ & Expt. & $\begin{array}{c}438 \mathrm{~K} \\
\text { Calc. } \\
\text { without IC }\end{array}$ & $\begin{array}{l}\text { Calc. } \\
\text { with IC }\end{array}$ \\
\hline I & 77(8) & $60.2(4)$ & 74.9(4) & 71(7) & $54.2(3)$ & $72.0(4)$ \\
\hline II & $10(2)$ & 12.1(1) & 12.1(1) & 11(2) & $12.4(1)$ & $12.4(1)$ \\
\hline III & 13(2) & $13.0(1)$ & $13.0(1)$ & $17(3)$ & $15.6(1)$ & $15.6(1)$ \\
\hline IV & $\ldots$ & $14.7(1)$ & $\ldots$ & $\ldots$ & $17.8(1)$ & $\ldots$ \\
\hline
\end{tabular}

${ }^{\mathrm{a}}$ Experimental populations obtained from the integrated $\nu(\mathrm{C}=\mathrm{O})$ bands of the infrared vibrational spectrum taken in a low-temperature neon matrix (see Ref. 22)

${ }^{\mathrm{b}}$ Populations calculated at thermal equilibrium using the torsional pathintegral Monte Carlo method with a MP2/6-311++ $\mathrm{G}^{* * *}$ torsional potentialenergy surface (see Ref. 26); values obtained by linear interpolation of calculations performed at 300,400 , and $500 \mathrm{~K}$.

${ }^{\mathrm{c}}$ Number in parentheses indicates the estimated error of the last reported digit. High-energy conformers not considered in this study are assumed to have interconverted to conformer I.

${ }^{\mathrm{d}}$ Calculated populations are reported with and without assuming the total interconversion (IC) of the conformer IV population to conformer I.

providing an equilibrium Boltzmann distribution of conformers and vibrational/rotational states. The molecules are then cooled for spectroscopic analysis via, for example, free-jet expansion in which collisions with the carrier gas quench the vibrational/rotational states of the sample..$^{36-38}$

The lower panel of Fig. 2 illustrates how the original conformer distribution in the oven can change during this cooling process. In the first case, the original distribution collapses vertically, preserving the conformer populations of the high-temperature equilibrium distribution. The molecules are thus vibrationally and rotationally cold but remain conformationally hot. This situation is ideal because it enables the observed populations to be related to the conformer thermodynamics of a known temperature. In the second case, some or all of the population of high-energy conformers in-
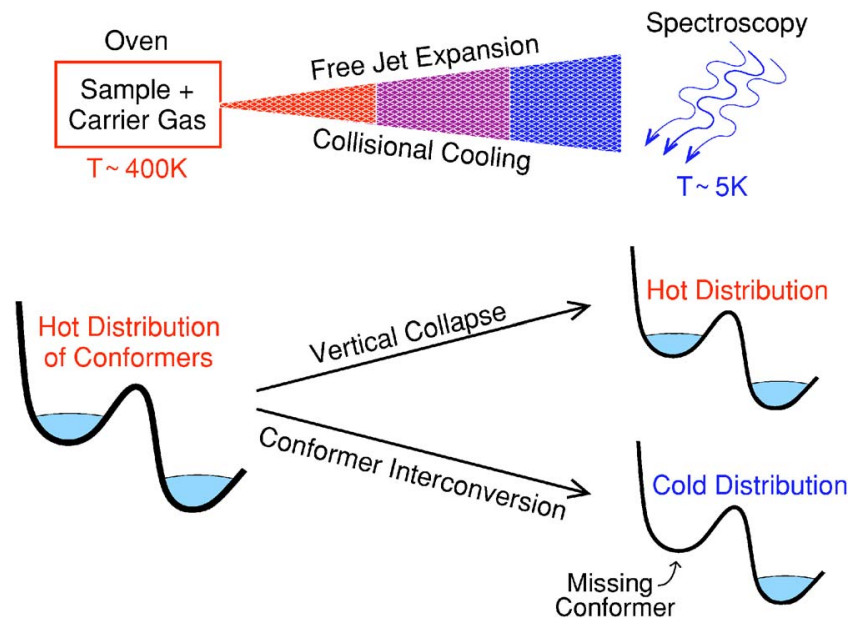

FIG. 2. Schematic illustration of conformer interconversion. The upper panel shows a typical experimental setup in which molecules are heated in an oven prior to free-jet expansion and spectroscopic analysis. The lower panel shows the corresponding distribution of conformers. During expansion, the distribution will either experience vertical collapse, preserving the population of high-energy conformers, or it will experience conformer interconversion and exhibit "missing" conformers.

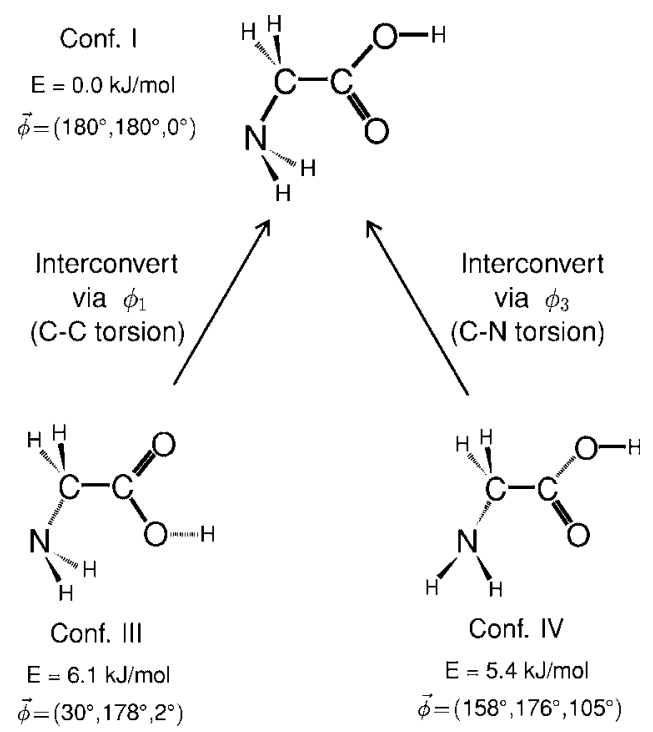

FIG. 3. Arrows indicate the two interconversion processes considered in this study. The torsional angles $\vec{\phi}=\left(\phi_{1}, \phi_{2}, \phi_{3}\right)$ correspond to intramolecular rotation about the $\mathrm{C}-\mathrm{C}$ bond, $\mathrm{C}-\mathrm{O}$ single bond, and $\mathrm{C}-\mathrm{N}$ bond, respectively. Energies determined by $\mathrm{CCSD}(\mathrm{T}) / \mathrm{MP} 2 / 6-311++\mathrm{G}^{* *}$ single-point calculations at MP2/6-311++ $\mathrm{G}^{* *}$-optimized geometries.

terconverts to low-energy conformers, distorting the thermodynamic data obtained from the experiment and giving rise to "missing" conformers.

The degree to which interconversion occurs during cooling is usually associated with the intramolecular potentialenergy surface of the molecule. ${ }^{17,32-35}$ Conformers with large barriers to interconversion are expected to be trapped during cooling and maintain their original, high-temperature population. On the other hand, conformers exhibiting low-energy pathways leading to more stable structures are more likely to be missing from the final spectrum. Implicit to this argument is the fact that, whereas the original distribution of molecules in the oven is a static property of the molecule, the theoretical description of the cooling process is a dynamical problem.

Application of this energy-barrier argument to the conformers of glycine does not seem to explain the fact that conformer III is observed experimentally but conformer IV is missing. By calculating $\operatorname{CCSD}(\mathrm{T}) / 6-311++\mathrm{G}^{* *}$ single-point energies at the optimized MP2/6-311+ $+\mathrm{G}^{* *}$ stationary points, we predict barriers of 3.1 and $2.8 \mathrm{~kJ} / \mathrm{mol}$ in the $\mathrm{III} \rightarrow \mathrm{I}$ and IV $\rightarrow \mathrm{I}$ interconversion pathways, respectively. The small difference in these barrier heights suggests that neither conformer would be substantially more prone to interconversion, so the elusiveness of conformer IV remains a mystery. Figure 3 shows that the interconversion pathways for conformers III and IV are largely associated with individual torsional motions. The III $\rightarrow$ I pathway corresponds to internal rotation about the $\mathrm{C}-\mathrm{C}$ bond ( $\phi_{1}$ torsion), and the $\mathrm{IV} \rightarrow \mathrm{I}$ pathway involves rotation about the $\mathrm{C}-\mathrm{N}$ bond $\left(\phi_{3}\right.$ torsion).

A previous study by Zhang et al. has considered the inelastic scattering of the glycine $\phi_{1}$ torsion in collisions 
with a hydrogen atom. ${ }^{39}$ It reported transition probabilities from the vibrational ground state of conformer I to excited vibrational states and found that the mean value of the $\phi_{1}$ torsional coordinate varied by up to $25^{\circ}$ in the potential well of conformer I. The possibility of conformer interconversion, however, was not addressed. Bowman and co-workers have used quantum scattering calculations to study the isomerization of HCN/HNC in collisions with an argon atom. ${ }^{40,41}$ These studies found relatively small isomerization probabilities that increased substantially for collision energies in excess of the isomerization potential-energy barrier.

We present quantum dynamical calculations of the collision-induced conformer interconversion of the glycine amino acid. We consider both the III $\rightarrow$ I and IV $\rightarrow$ I interconversion processes for collisions involving the $\mathrm{He}, \mathrm{Ne}$, and $\mathrm{Ar}$ rare-gas atoms. Although the single-collision processes considered here constitute only a preliminary description of molecular cooling via free-jet expansion or substrate deposition, it is the first quantum-mechanical study of collision-induced conformational changes in a biomolecule of which the authors are aware. Section II presents the scattering theory that we employ in this study, and Sec. III presents the details of our calculations. Finally, Sec. IV presents the results of our calculations and a subsequent discussion.

\section{THEORY}

In our calculations, we use the torsional close-coupling rotational infinite order sudden (TCC-IOS) method. Since we have previously used this approach to study torsional dynamics, ${ }^{42}$ only the most important details will be highlighted here. For further information, the reader is referred to Ref. 42.

The colliding rare-gas atom has spherical polar coordinates $(R, \Theta, \Phi)$ with respect to the midpoint of the $\mathrm{C}-\mathrm{C}$ bond in the glycine molecule. The $\mathrm{C}-\mathrm{C}$ bond is taken to be the $z$ axis of our coordinate with the $\mathrm{N}$ atom lying in the $(+,+)$ quadrant of the $x z$ plane. The $y$ axis is chosen to obtain a right-handed coordinate system. In our TCC-IOS calculations we assume that the rotation of the target molecule is much slower than the time scale of the collision. Therefore, the infinite order sudden approximation is valid, and we can investigate the collision between glycine and a rare-gas atom for each initial collision geometry $(\Theta, \Phi)$ separately. Subsequent averaging over all angles $(\Theta, \Phi)$ yields total-energytransfer probabilities.

Only one torsion at a time is explicitly treated in our scattering calculations. The one-dimensional Hamiltonian for this torsion is

$$
\hat{H}_{j}=-\frac{\hbar^{2}}{2 I_{j}} \frac{d^{2}}{d \phi_{j}^{2}}+v_{j}\left(\phi_{j}\right)
$$

where $v_{j}\left(\phi_{j}\right)$ is the torsional potential-energy surface and the torsional moment of inertia $I_{j}$ is defined as in Ref. 42. Diagonalizing $\hat{H}_{j}$ in a suitable basis yields a set of eigenfunctions $\left\{h_{i}\left(\phi_{j}\right)\right\}$ and energies $\left\{E_{i}^{j}\right\}$ for each torsional mode $j$. See Sec. III A for the details of this calculation.
The total wave function, $\Psi\left(R, \phi_{j}, t ; \Theta, \Phi\right)$, is subsequently expanded in terms of $\left\{h_{i}\left(\phi_{j}\right)\right\}$ as

$$
\Psi\left(R, \phi_{j}, t ; \Theta, \Phi\right)=\sum_{\lambda}^{N_{R}} \sum_{i}^{N_{j}} c_{\lambda i}(t ; \Theta, \Phi) s_{\lambda}^{-}(R) h_{i}\left(\phi_{j}\right),
$$

where $s_{\lambda}^{-}(R)$ are "wrapped" sinc-discrete variable representation (DVR) basis functions. ${ }^{43-47}$ These functions are nonzero only at the grid points $R_{\lambda}$, where $R_{\lambda}=\lambda * \Delta+R_{\text {min }}$ with $\Delta$ the grid spacing and $R_{\min }$ the minimum scattering distance.

The total Hamiltonian for this system is defined as

$$
\hat{H}=-\frac{\hbar^{2}}{2 \mu} \frac{\partial^{2}}{\partial R^{2}}+\hat{H}_{j}+V_{I}\left(R, \phi_{j} ; \Theta, \Phi\right) \text {. }
$$

Inserting Eqs. (2) and (3) into the time-dependent Schrödinger equation, $i \hbar \partial \Psi / \partial t=\hat{H} \Psi$, and multiplying by the basis from the left, we get the following equations of motion:

$$
\begin{aligned}
i \hbar \frac{\partial c_{\lambda i}(t ; \Theta, \Phi)}{\partial t}= & \sum_{\lambda^{\prime}}^{N_{\lambda}} T_{\lambda \lambda^{\prime}}^{R} c_{\lambda^{\prime} i}(t ; \Theta, \Phi) \\
& +\sum_{i^{\prime}}^{N_{j}} B_{i i^{\prime}}\left(R_{\lambda} ; \Theta, \Phi\right) c_{\lambda i^{\prime}}(t ; \Theta, \Phi) .
\end{aligned}
$$

Here,

$$
T_{\lambda \lambda^{\prime}}^{R}=-\frac{\hbar^{2}}{2 \mu}\left\langle s_{\lambda}^{-}(R)\left|\frac{\partial^{2}}{\partial R^{2}}\right| s_{\lambda^{\prime}}^{-}(R)\right\rangle
$$

and

$$
B_{i i^{\prime}}(R ; \Theta, \Phi)=\left\langle h_{i}\left(\phi_{j}\right)\left|V_{I}\left(R, \phi_{j} ; \Theta, \Phi\right)\right| h_{i^{\prime}}\left(\phi_{j}\right)\right\rangle+E_{i}^{j} \delta_{i i^{\prime}},
$$

where the close-coupling matrix elements are evaluated using the same quadrature as used to obtain $E_{i}^{j}$.

The solution of the equations of motion in time gives us the probabilities $P_{i \rightarrow i^{\prime}}^{j}(E ; \Theta, \Phi)$ (see Sec. III D) for collisioninduced transition between torsional states $i$ and $i^{\prime}$ as a function of the total energy $E$. The initial collision energy is given from $E$ as $E-E_{i}^{j}$. From $P_{i \rightarrow i^{\prime}}^{j}(E ; \Theta, \Phi)$ we can calculate average probabilities via

$$
P_{i \rightarrow i^{\prime}}^{j}(E)=\frac{1}{4 \pi} \int_{0}^{\pi} d \Theta \int_{0}^{2 \pi} d \Phi P_{i \rightarrow i^{\prime}}^{j}(E ; \Theta, \Phi) \sin \Theta .
$$

\section{COMPUTATIONAL DETAILS}

\section{A. Torsional potential and eigenstates}

We have previously reported a torsional potential-energy surface $V\left(\phi_{1}, \phi_{2}, \phi_{3}\right)$ for glycine based on the energies for a $12 \times 12 \times 12$ grid of torsional angle values. ${ }^{26}$ The energy of each grid point was calculated at the MP2/6-311++ $\mathrm{G}^{* *}$ level of theory, accounting for the relaxation of the nontorsional degrees of freedom. In the current study, onedimensional (1D) torsional potential-energy surfaces $v_{1}\left(\phi_{1}\right)$ 
TABLE II. Fitting parameter $a_{j, n}\left(\right.$ a.u. $\left.\times 10^{-6}\right)$ for the $1 \mathrm{D}$ torsional potentials in Eq. (8).

\begin{tabular}{rrr}
\hline \hline & $\mathrm{III} \rightarrow \mathrm{I}$ & $\mathrm{IV} \rightarrow 1$ \\
$j$ & $j=1$ & $j=3$ \\
\hline 0 & 4616.8 & 6021.37 \\
1 & 762.1 & -2377.07 \\
2 & -1144.9 & 649.61 \\
3 & 499.8 & -1455.47 \\
4 & 52.3 & 43.52 \\
5 & 13.5 & 76.64 \\
6 & 53.6 & 62.00 \\
7 & -3.1 & -19.01 \\
8 & 1.1 & 12.99 \\
9 & -1.8 & -3.88
\end{tabular}

Fitting error

(Avg. rms)

1.1

0.54

and $v_{3}\left(\phi_{3}\right)$ were obtained from $V\left(\phi_{1}, \phi_{2}, \phi_{3}\right)$ by calculating the minimum-energy pathways for III $\rightarrow$ I and IV $\rightarrow$ I interconversions, respectively. The pathways were calculated at 360 points along the corresponding torsional coordinate, and the points were fitted using periodic functions of the form

$$
v_{j}\left(\phi_{j}\right)=\frac{a_{j, 0}}{2}+\sum_{n=1}^{9} a_{j, n} \cos \left(n \phi_{j}\right), \quad j=1,3 .
$$

The fitting parameters $a_{j, n}$ are reported in Table II. The conformer interconversion barriers calculated using the $v_{j}\left(\phi_{j}\right)$ agree well with the reference $\operatorname{CCSD}(\mathrm{T})$ calculations reported in the Introduction. Specifically, for the III $\rightarrow \mathrm{I}[\mathrm{IV} \rightarrow \mathrm{I}]$ interconversion pathway, the $\operatorname{CCSD}(\mathrm{T}) / 6-311++\mathrm{G}^{* *} / /$ MP2/6-311 $++\mathrm{G}^{* *}$ value of 3.1 [2.8] $\mathrm{kJ} / \mathrm{mol}$ for the barrier height agrees well with the $v_{1}\left[v_{3}\right]$ value of $3.2[3.0] \mathrm{kJ} / \mathrm{mol}$.
For each torsion, the potential $v_{j}\left(\phi_{j}\right)$ in Eq. (8) is used to construct the 1D Hamiltonian $\hat{H}_{j}$ in Eq. (1). The moment of inertia values of $I_{1}=123691.0$ a.u. and $I_{3}=11652.3$ a.u. is employed as in the previous work. ${ }^{26}$ Eigensolutions of the $\hat{H}_{j}$ are obtained by diagonalization in the basis of functions $(1 / \sqrt{2 \pi}) e^{ \pm i k \phi}, k=0,1, \ldots, 1000$. Figure 4 presents the $1 \mathrm{D}$ torsional potential curves [Eq. (8)] and their corresponding eigenfunctions. Each eigenfunction plotted in the figure is vertically translated according to its eigenvalue.

The potential curve for the $\mathrm{C}-\mathrm{C}$ torsion (Fig. 4, left panel) has minima at $\phi_{1}=180^{\circ}$ for conformer $\mathrm{I}$ and at $\phi_{1}= \pm 30^{\circ}$ for the symmetric, nonplanar geometries of conformer III. This torsional potential curve differs from the one presented in Ref. 39 in two ways: it exhibits double-well character for conformer III and has a substantially lower barrier to interconversion $(3 \mathrm{~kJ} / \mathrm{mol}$ vs $3 \mathrm{kcal} / \mathrm{mol})$. The differences are attributed to the fact that the potential curve presented in the current study is calculated at a different level of theory, employing a substantially larger basis set, and accounts for the adiabatic relaxation of the rest of the molecule as a function of the torsional coordinate. The relatively small barrier that we calculate for $\mathrm{III} \rightarrow \mathrm{I}$ interconversion is more consistent with the upper-bound estimate of $5.8 \mathrm{~kJ} / \mathrm{mol} \mathrm{ob}-$ tained from matrix annealing experiments. ${ }^{18}$ The $\mathrm{C}-\mathrm{N}$ torsional potential curve (right panel) has minima at $\phi_{3}=0^{\circ}$ for conformer I and at $\phi_{3}= \pm 105^{\circ}$ for the symmetric, nonplanar geometries of conformer IV. Comparison of the potential curves shown in Fig. 4 reiterates the fact that the torsional barrier for interconversion from the higher-energy conformers (III or IV) to conformer I is almost identical.

Despite the basic similarities of the two potential curves in Fig. 4, the torsional eigenfunctions differ substantially. The moment of inertia for the $\mathrm{C}-\mathrm{N}$ torsion $\left(I_{3}\right)$ is approximately an order of magnitude smaller than that of the $\mathrm{C}-\mathrm{C}$ torsion $\left(I_{1}\right)$, which contributes to larger spacing between the
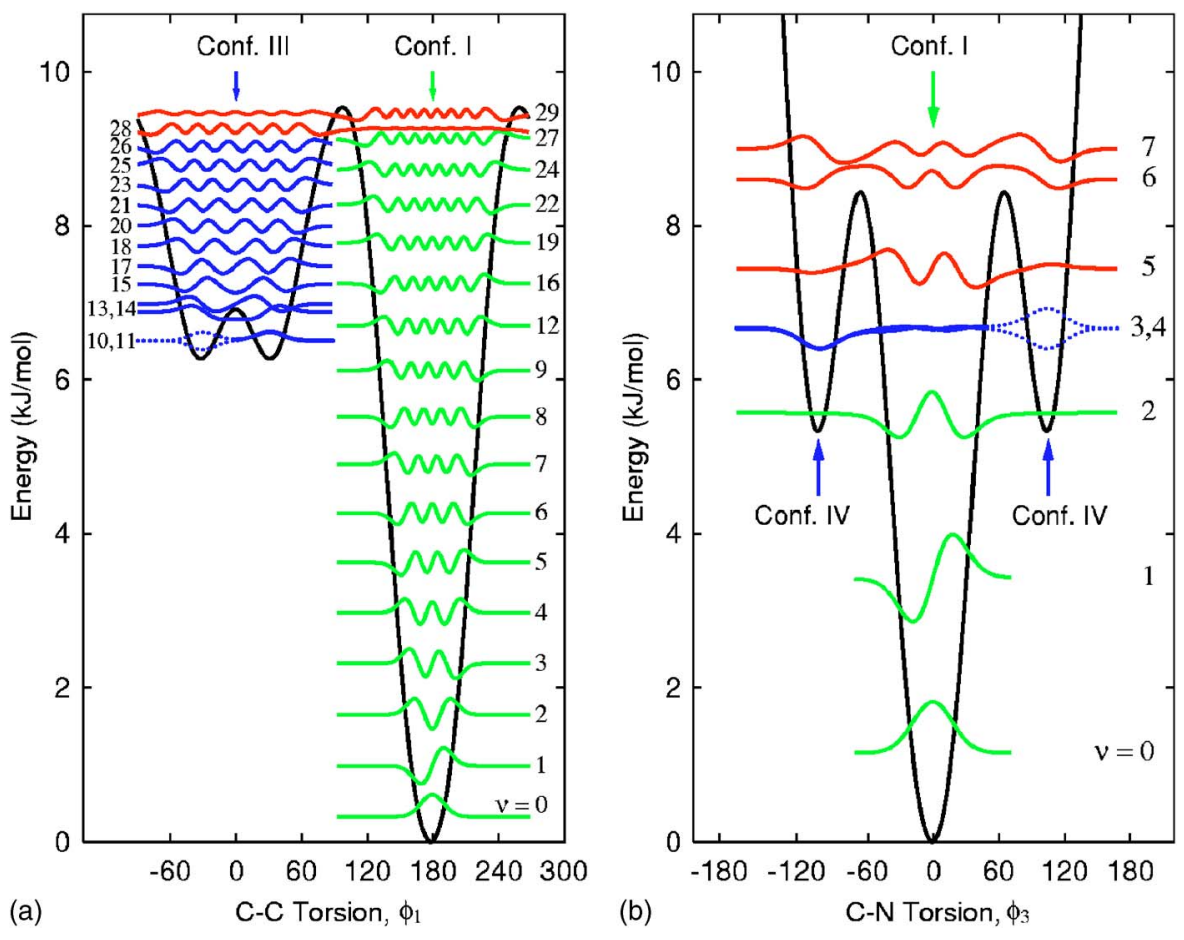

FIG. 4. Potential surfaces $v_{j}\left(\phi_{j}\right)$ and vibrational eigenfunctions for the $\mathrm{C}-\mathrm{C}$ torsion $\phi_{1}$ (left panel) and the $\mathrm{C}-\mathrm{N}$ torsion $\phi_{3}$ (right panel). Eigenfunctions plotted in red $v_{28}$ and $v_{29}$ in the left panel and $v_{5}-v_{7}$ in the right panel) have nonzero amplitude corresponding to both conformers. 
$\mathrm{C}-\mathrm{N}$ torsional eigenvalues and a greater degree of tunneling in the corresponding eigenfunctions. Furthermore, we note that the torsional barrier to IV $\rightarrow$ I conformer interconversion is narrow in comparison to that for $\mathrm{III} \rightarrow \mathrm{I}$ interconversion. Eigenfunctions exhibiting amplitude in both conformer wells (plotted in red) are found only at the very top of the interconversion barrier in the left panel, whereas they occur deeper in the conformer wells of the right panel.

\section{B. Definition of conformer population}

To simulate collision-induced conformer interconversion, we consider vibrationally inelastic scattering from the ground state of the higher-energy conformer. For example, in studying the $\mathrm{III} \rightarrow \mathrm{I}$ interconversion process, the glycine molecule is prepared in the $\nu=10$ vibrational state (see Fig. 4), and the angle-averaged transition probabilities $P_{10 \rightarrow i^{\prime}}^{1}(E)$ are calculated for $i^{\prime}=0,1, \ldots, 34$. Likewise, for the IV $\rightarrow$ I interconversion process, the molecule is prepared in the $\nu=3$ state, and the $P_{3 \rightarrow i^{\prime}}^{3}(E)$ are calculated for $i^{\prime}=0,1, \ldots, 10$. Fewer states are needed to describe IV $\rightarrow$ I interconversion because of the larger spacing of the eigenvalues.

Having obtained the transition probabilities, we relate them to the postcollision torsional distribution

$$
\rho_{j}\left(\phi_{j}, E\right)=\sum_{i^{\prime}=0}^{N_{j}}\left|h_{i^{\prime}}\left(\phi_{j}\right)\right|^{2} P_{i \rightarrow i^{\prime}}^{j}(E),
$$

where $j$ indicates the torsion under consideration, $N_{j}$ is the number of vibrational states included in the scattering calculation, and $i$ is the ground state of the preinterconversion conformer. As was previously stated, if $j=1$, then $N_{j}=34$ and $i=10$, and if $j=3$, then $N_{j}=10$ and $i=3$.

The torsional distribution is integrated over the range of angles associated with conformer I to yield the total collision-induced probability of interconversion,

$$
P_{j}^{\mathrm{TIC}}(E)=\int_{A}^{B} d \phi_{j} \rho_{j}\left(\phi_{j}, E\right)-C_{j},
$$

where the integration range corresponds to the positions of the torsional interconversion barrier peaks. That is, when $j=1, \quad[A, B]=\left[98^{\circ}, 262^{\circ}\right], \quad$ and when $j=3, \quad[A, B]$ $=\left[-66^{\circ}, 66^{\circ}\right]$ (see Fig. 4). The constant in Eq. (10),

$$
C_{j}=\int_{A}^{B} d \phi_{j}\left|h_{i}\left(\phi_{j}\right)\right|^{2},
$$

ensures that in the absence of a collision, the total probability of interconversion is zero. This term is necessary because the initial $\mathrm{C}-\mathrm{N}$ torsional eigenfunction $h_{3}\left(\phi_{3}\right)$ has about $2 \%$ of its probability distribution in conformer I. Finally, we note that with the use of angle-dependent transition probabilities $P_{i \rightarrow i^{\prime}}^{j}(E ; \Theta, \Phi)$ in Eq. (9), it is also straightforward to calculate the angle-dependent torsional distribution $\rho_{j}\left(\phi_{j}, E ; \Theta, \Phi\right)$ and the angle-dependent total probability of interconversion $P_{j}^{\mathrm{TIC}}(E ; \Theta, \Phi)$.
Clearly, Eq. (10) is not the only possible definition of interconversion probability. We employ it because it is convenient and intuitive, but we also note that this definition allows high-energy vibrational states, which are not localized in either conformer well, to contribute to the calculated probability of interconversion. In reality, the degree to which these excited states are quenched into conformer I by subsequent collisions is a dynamical problem not addressed in the current study.

As an alternative definition, we introduce the "direct" interconversion probability

$$
P_{j}^{\mathrm{DIC}}(E)=\sum_{i^{\prime}=0}^{i-1} P_{i \rightarrow i^{\prime}}^{j}(E),
$$

which only includes contributions to interconversion arising from quenching transitions $\left(i^{\prime}<i\right)$. It is evident from Fig. 4 that the lower-energy states included in direct interconversion are localized almost entirely in the conformer I potential well. A similar definition was used to describe the isomerization of HCN/HNC by Lan and Bowman. ${ }^{40}$ Both the total interconversion probability $P_{j}^{\mathrm{TIC}}(E)$ and the more stringently defined direct interconversion probability $P_{j}^{\mathrm{DIC}}(E)$ are considered in this study.

\section{Interaction potential}

As in previous studies, ${ }^{42,48}$ the potential of interaction $V_{I}$ between the colliding rare-gas atom ( $\mathrm{He}, \mathrm{Ne}$, or $\mathrm{Ar}$ ) and the atoms of glycine $(\mathrm{H}, \mathrm{C}, \mathrm{N}$, and $\mathrm{O})$ were described using simple, pairwise expansions of the form

$$
V_{I}=\sum_{i=1}^{N} V_{i}\left(R_{i}\right),
$$

where $R_{i}$ is the distance from the rare-gas atom to the glycine atom $i$ and $N$ is the number of atoms in glycine. For the helium collisions, the pair-interaction function $V_{i}\left(R_{i}\right)$ was calculated using

$$
V_{i}\left(R_{i}\right)=A_{i} \exp \left(-B_{i} R_{i}\right),
$$

with parameters $\left(A=8.00 E_{h}\right.$ and $\left.B=2.33 a_{0}\right)$ when $i$ is a $\mathrm{H}$ atom and $\left(A=39.92 E_{h}\right.$ and $\left.B=2.01 a_{0}\right)$ otherwise. $^{49}$ Longrange van der Waals attractions between the He and glycine atoms are expected to be negligible over the range of collision energies considered here.

For calculations involving the more polarizable $\mathrm{Ne}$ and Ar atoms, van der Waals interactions were included via the exponential-6 functional form

$$
V(R)=\epsilon\left[6 \exp [\alpha(1-x)]-\alpha x^{-6}\right] /(\alpha-6),
$$

where $x=R / R_{e}$ and with parameters $\epsilon, R_{e}$, and $\alpha$ listed inTable III. These values were obtained using the prescription described in Ref. 50 with updated experimental data. ${ }^{51,52}$

\section{Wave-packet calculations}

The wave-packet calculations presented here were performed in a similar fashion to the calculations in Ref. 42. We propagated an initial wave packet of the form 
TABLE III. Parameters used for Ne-glycine and Ar-glycine interaction potentials [Eq. (15)].

\begin{tabular}{lccc}
\hline \hline & $R_{e} / a_{0}$ & $\epsilon / 10^{-6} E_{h}$ & $\alpha$ \\
\hline $\mathrm{Ne}-\{\mathrm{C}, \mathrm{N}, \mathrm{O}\}$ & 5.84 & 134 & 13.0 \\
$\mathrm{Ne}-\mathrm{H}$ & 6.15 & 64 & 14.3 \\
$\mathrm{Ar}-\{\mathrm{C}, \mathrm{N}, \mathrm{O}\}$ & 6.58 & 221 & 14.6 \\
$\mathrm{Ar}-\mathrm{H}$ & 6.75 & 116 & 15.1 \\
\hline \hline
\end{tabular}

$$
G(R)=\frac{1}{\sqrt[4]{2 \pi \beta}} \exp \left(-i k_{0} R\right) \exp \left[\frac{-\left(R-R_{0}\right)^{2}}{4 \beta}\right],
$$

using a ( $m=6$ and $n=4)$ symplectic integrator. ${ }^{53}$ During the calculations, the wave function was absorbed at the edges of the grid to avoid unphysical reflections using a damping function of the form $\exp \left(-\alpha\left(R-R_{a}\right)^{2}\right){ }^{54}$ To cut down on the size of the calculation, we used a grid point selection scheme similar to the one in Ref. 55. Thus, we restricted our calculation to the area of the grid where the potential lay between $V_{c}^{(i)}$ at small $R$ and $V_{c}^{(o)}$ at large $R$. Starting positions $R_{0}$ and absorption regions $R_{a}$ were shifted accordingly to take account of this. All calculations were performed on a Pentium IV processor using the GOTO BLAS (Ref. 56) for the matrixvector multiplications and FFTW (Refs. 57-59) for the fast Fourier transforms.

The asymptotic analysis of the time-dependent calculations was done in two ways. First, we calculated energydependent reaction probabilities via the flux through a surface at a distance $R_{f}{ }^{60}$ Secondly, we used the damping for the wave packet to calculate transition probabilities through a wave packet splitting technique. ${ }^{61,62}$ The use of both methods enabled us to check the convergence of the reaction probabilities.

The latter transition probabilities were also employed to calculate the collision times reported in this article. We use the definition of collision time provided in Ref. 42. See Table IV for a summary of the parameters used in the wave-packet calculations.

\section{E. Infrared spectrum calculations}

To construct the IR spectra presented in Sec. IV C, vibrational frequency calculations including anharmonic cor- rections were performed at the MP2/6-311++ $\mathrm{G}^{* *}$ level of theory for conformers I-IV of glycine. All electronic structure calculations presented in the current paper were performed using the GAUSSIAN03 package. ${ }^{63}$ The spectra for the individual conformers were generated from the calculated anharmonic frequencies and the harmonic absorption intensities using Lorentzian functions with a full width half maximum of $5 \mathrm{~cm}^{-1}$. Only the fundamental bands were included in the generation of these spectra.

By virtue of Beer's law, the combined absorption spectrum for a distribution of conformers can be constructed from a weighted sum of the individual conformer spectra, where the weighting factor for the spectrum of each conformer is the same as the population of that conformer in the distribution. That is, the IR spectrum corresponding to the equilibrium distribution of conformers at $0 \mathrm{~K}$ will simply be the same as the spectrum of the lowest-energy conformer. However, a spectrum corresponding to a more complicated distribution of conformers will be a linear combination of the individual conformer spectra. In Sec. IV C, we present spectra for two possible distributions of the glycine conformers at $438 \mathrm{~K}$, where the conformer populations (and thus the weighting factors for the individual spectra) are calculated using the torsional path-integral Monte Carlo (TPIMC) method. ${ }^{26}$

\section{RESULTS AND DISCUSSION}

\section{A. Scattering calculation results}

Figure 5 presents the calculated probabilities of III $\rightarrow$ I and IV $\rightarrow$ I conformer interconversions due to collisions involving the $\mathrm{He}, \mathrm{Ne}$, and $\mathrm{Ar}$ rare-gas atoms. These total probabilities $P_{j}^{\mathrm{TIC}}(E)$, which are defined in Eq. (10), are plotted as a function of the energy of the collision (i.e., the initial translational energy of the rare-gas atom). The vertical lines indicate the torsional potential-energy barrier for the respective interconversion processes.

TABLE IV. Various parameters for the wave-packet calculations. All values are in a.u. Value $(X)$ is the value for the calculations involving rare-gas atom $X$.

\begin{tabular}{ccccc}
\hline \hline Variable & Explanation & Value $(\mathrm{He})$ & Value $(\mathrm{Ne})$ & Value (Ar) \\
\hline$\beta$ & Width of initial wave packet & 0.085 & 0.05 & 0.05 \\
$R_{0}$ & Center of initial wave packet & 40.0 & 41.0 & 41.0 \\
$E_{0}$ & Initial energy of wave packet & 0.005 & 0.005 & 0.005 \\
$N_{R}$ & Number of grid points in $R$ coordinate & 600 & 600 & 600 \\
$R_{\min }$ & Minimum scattering distance & 0.0 & 0.0 & 0.0 \\
$R_{\max }$ & Maximum scattering distance & 50.0 & 50.0 & 50.0 \\
$\Delta t$ & Time step in propagation & 20.0 & 20.0 & 20.0 \\
$R_{f}$ & Scattering distance for flux analysis & 42.4 & 45.9 & 45.9 \\
$R_{a}$ & Scattering absorption distance & 43.0 & 46.0 & 46.0 \\
$\alpha$ & Strength of absorption & 0.0015 & 0.005 & 0.005 \\
$V_{c}^{(i)}$ & Potential cutoff for inner edge grid & 0.3 & 0.3 & 0.3 \\
$V_{c}^{(o)}$ & Potential cutoff for outer edge grid & $1.0 \times 10^{-6}$ & $1.0 \times 10^{-6}$ & $1.0 \times 10^{-6}$ \\
\hline \hline
\end{tabular}



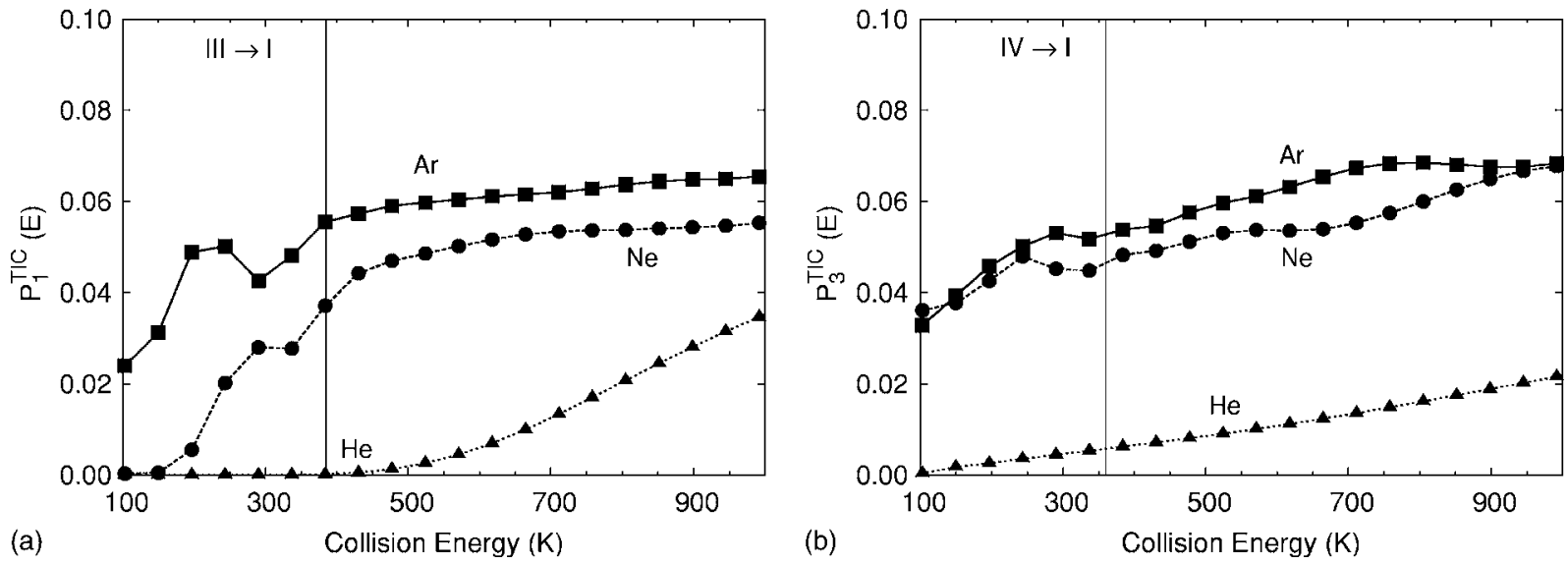

FIG. 5. Angle-averaged total interconversion probabilities calculated for the (a) III $\rightarrow$ I and (b) IV $\rightarrow$ I processes as a function of collision energy. Results shown for collisions involving the $\mathrm{He}, \mathrm{Ne}$, and $\mathrm{Ar}$ rare-gas atoms. The vertical line indicates the energy of the torsional barrier to interconversion.

An important initial observation from Fig. 5 is that interconversion actually does occur. Substantial Ne- and Arinduced interconversion probabilities are calculated for both processes, particularly for collision energies in excess of the torsional barrier. The He-induced interconversion probabilities show similar dependence on collision energy but are consistently smaller in magnitude.

Figure 6 shows that when the direct definition of conformer interconversion is employed [Eq. (12)], similar trends emerge. Conformer interconversion remains strongest for collisions involving the $\mathrm{Ne}$ and Ar rare-gas atoms, and neither III $\rightarrow$ I nor IV $\rightarrow$ I interconversion appears to be markedly favored, particularly for collision energies in excess of the torsional barrier. Because the direct interconversion probability only includes contributions from quenching transitions, it is expected that the magnitude of the probabilities in Fig. 6 is lower than those in Fig. 5. However, the pronounced $\mathrm{III} \rightarrow$ I probability peaks at 300 and $250 \mathrm{~K}$ for the $\mathrm{Ne}$ and $\mathrm{Ar}$ collisions, respectively, are interesting and unexpected features of Fig. 6(a). These peaks resemble quantummechanical resonances between vibrational states, but inspection of the individual state-to-state transition probabilities reveals no dominant contribution. We will consider the origin of this low-energy interconversion probability later in Sec. IV B.

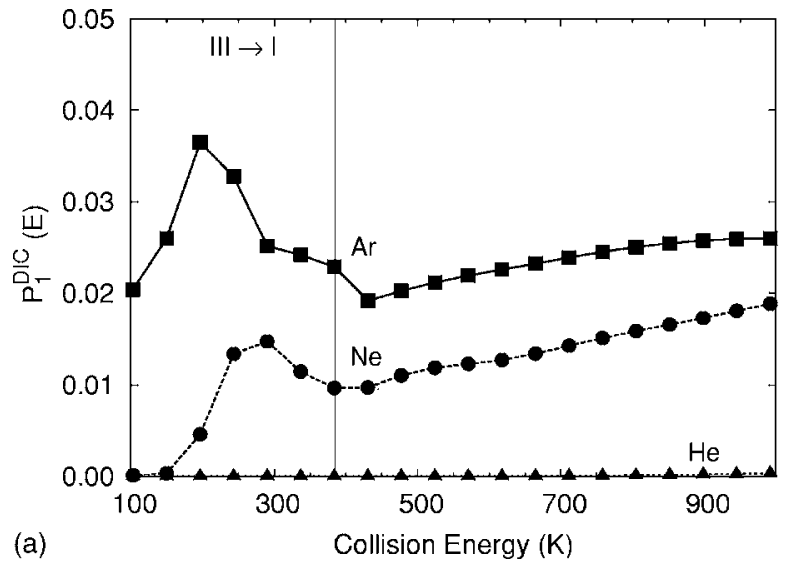

A more detailed picture of conformer interconversion is available from the angle-dependent interconversion probabilities $P_{j}^{\mathrm{TIC}}(E ; \Theta, \Phi)$ for Ne-glycine collisions at $290 \mathrm{~K}$ [Fig. 7(a)] and $430 \mathrm{~K}$ [Fig. 7(b)]. These probability plots show that only a few collision angles contribute to the angleaveraged results in Figs. 5 and 6 . However, for the active angles, large probabilities of over $50 \%$ are found for both interconversion processes.

At both collision energies shown in Fig. 7, the IV $\rightarrow$ I interconversion probability (right column) has distinct peaks around collision angles $(\cos \Theta, \Phi)=\left(0.8,20^{\circ}\right)$ and $(0.2$, $\left.60^{\circ}\right)$. The molecular illustrations show that these collision angles correspond to glancing blows of the rare-gas atom against the hydrogen atoms on the amine group. In each picture, the stricken hydrogen atom is colored black. The twisting motion of this amine group corresponds to the IV $\rightarrow$ I interconversion coordinate (see Fig. 3), so it is natural that excitation of this mode should lead to large interconversion probabilities.

A similar ballistic interpretation helps to explain the III $\rightarrow$ I interconversion probabilities in the left column of Fig. 7. At a collision energy of $430-\mathrm{K}$, the probability has distinct peaks which correspond to glancing blows against the two carbonyl oxygens (at $\Phi=0^{\circ}$ and $\Phi=180^{\circ}$ ) as well as a peak

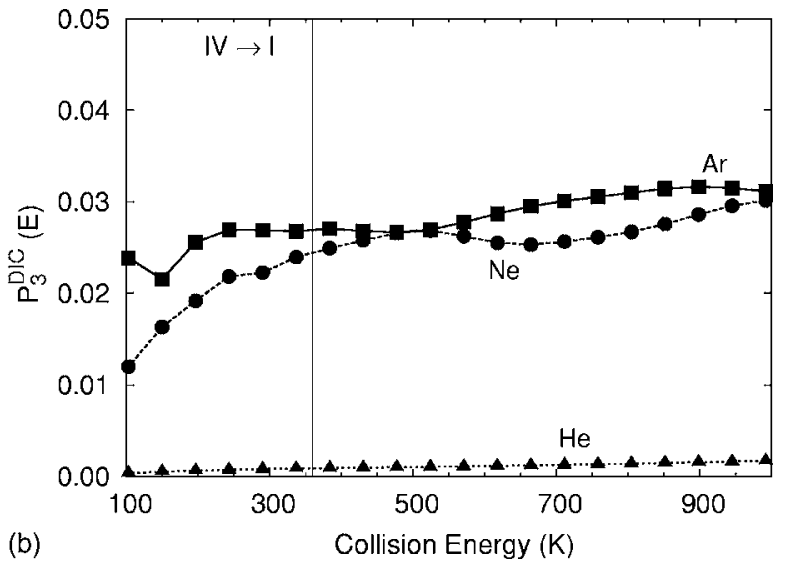

FIG. 6. Angle-averaged direct interconversion probabilities calculated for the (a) III $\rightarrow$ I and (b) IV $\rightarrow$ I processes as a function of collision energy. Results shown for collisions involving the $\mathrm{He}, \mathrm{Ne}$, and Ar rare-gas atoms. The vertical line indicates the energy of the torsional barrier to interconversion. 

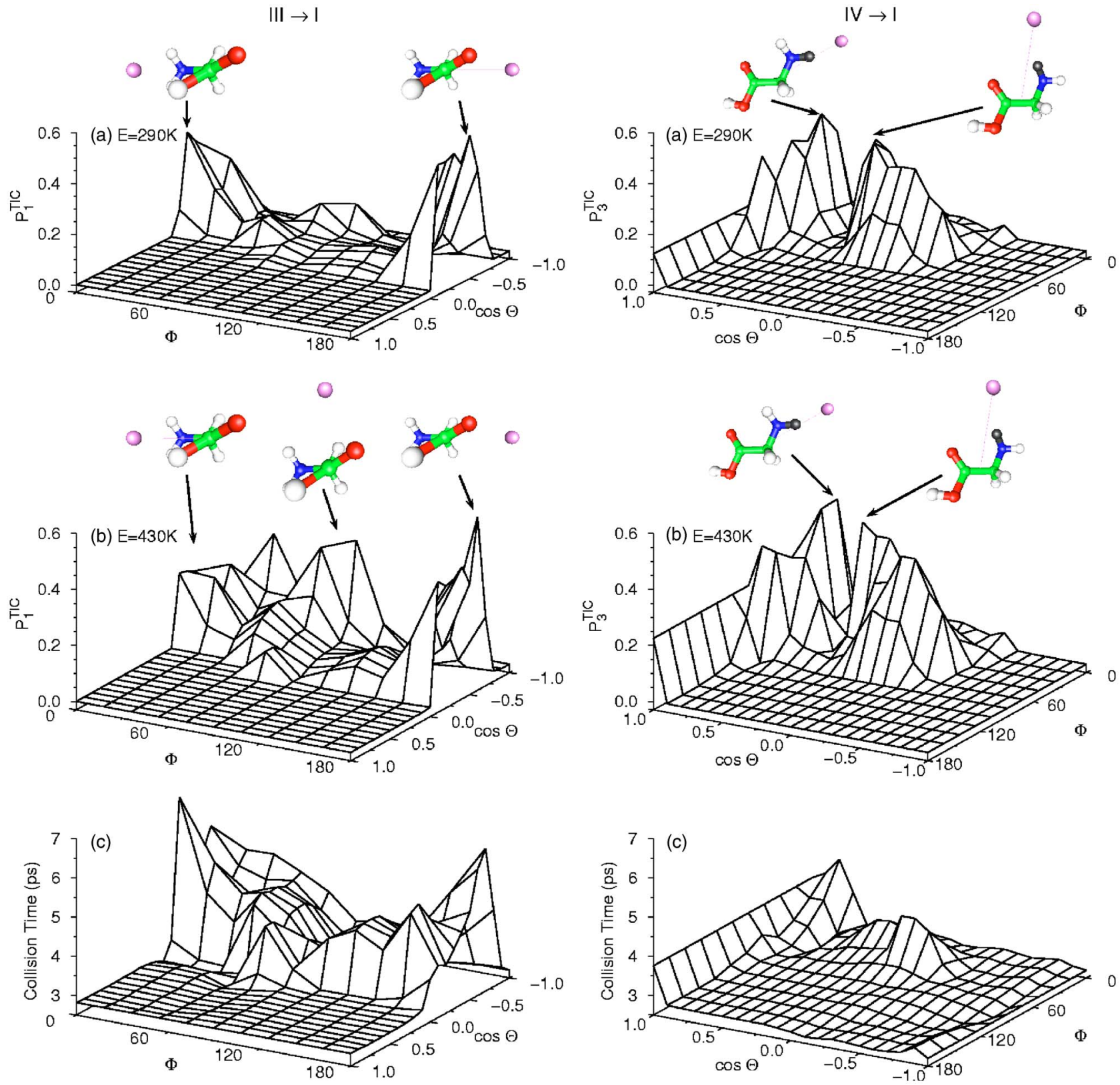

FIG. 7. Angle-dependent total interconversion probabilities for Ne-glycine collisions at energy (a) $290 \mathrm{~K}$ and (b) $430 \mathrm{~K}$. Results for the III $\rightarrow \mathrm{I}$ process are in the left column; results for the IV $\rightarrow$ I process are in the right column. The molecular diagrams illustrate collision angles corresponding to large interconversion probability. Part (c) presents the calculated collision times for the two interconversion processes.

at $\Phi=90^{\circ}$ which corresponds to a perpendicular blow to the carbonyl group. The probability peak for the perpendicular collision $\left(\Phi=90^{\circ}\right)$ disappears in the lower-energy collisions, whereas the other peaks remain relatively unchanged.

The angle dependence of the calculated collision times in Fig. 7(c) clearly correlates with that of the probabilities in Figs. 7(a) and 7(b). Longer collision times are generally calculated at angles leading to interconversion. However, we also note that the length of the collision time appears to correlate with the type of collision that occurs. For the $\mathrm{III} \rightarrow \mathrm{I}$ interconversion process, the collision time is much longer (6-7 ps) for collisions at $\Phi=0^{\circ}$ and $\Phi=180^{\circ}$ (which cause interconversion at both 290 and $430 \mathrm{~K}$ ) than at $\Phi=90^{\circ}$ (which only cause interconversion at the higher energy). No evidence of long collision times is seen for the
$\mathrm{IV} \rightarrow \mathrm{I}$ interconversion process, indicating that fast collisions taking less than 4 ps occur at both 290 and $430 \mathrm{~K}$.

To explore whether our calculations of collision-induced conformer interconversion are most sensitive to either (a) the mass of the rare-gas atom or (b) the potential energy of interaction between the rare-gas atom and the glycine molecule, we reconsider the angle-averaged total probability of IV $\rightarrow$ I interconversion in Fig. 8. Figure 8(a) presents interconversion probabilities for the various interaction potentials, assuming in each case that the mass of the colliding atom is equal to that of the He atom. In Fig. 8(b), the results for the three interaction potentials are again presented, but the mass of the colliding atom is set to that of the $\mathrm{Ne}$ atom. Comparison of Figs. 8(a) and 8(b) to each other and to Fig. 5(b) clearly reveals that the mass of the colliding rare- 

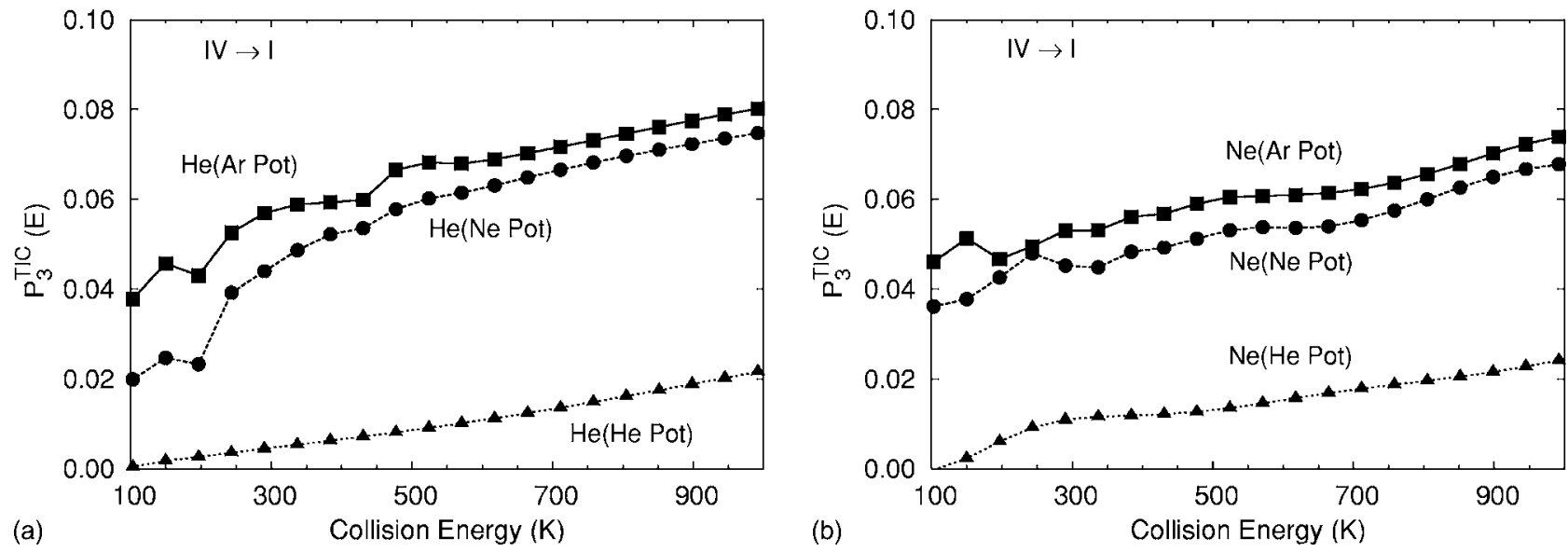

FIG. 8. Angle-averaged total interconversion probabilities calculated for the IV $\rightarrow$ I process as a function of energy. In (a), the colliding atom mass is set to that of $\mathrm{He}$ for all three interaction potentials. In (b), the colliding atom mass is set to that of Ne for all three interaction potentials. The agreement between the two plots indicates that the interconversion probability is more sensitive to the interaction potential than the mass of the colliding atom.

gas atom has little impact on the IV $\rightarrow$ I conformer interconversion. The degree of interconversion is much more dependent on the interaction potential of the colliding atom, with the weakly attractive interaction potentials of the $\mathrm{Ne}$ and $\mathrm{Ar}$ atoms causing more interconversion than the purely repulsive potential of the He atom.

\section{B. Conformer interconversion mechanisms}

Comparison of the results in Figs. 5-7 reveals an interesting difference in the $\mathrm{III} \rightarrow \mathrm{I}$ and $\mathrm{IV} \rightarrow \mathrm{I}$ interconversion processes: The calculated III $\rightarrow$ I probabilities appear to be sensitive to whether the collision energy is higher or lower than the torsional potential-energy barrier, whereas the IV $\rightarrow$ I interconversion probabilities do not. In Fig. 5(a), and even more dramatically in Fig. 6(a), the energy dependence of the interconversion probability is very different for collisions with energy higher and lower than that indicated by the vertical line. No such change in energy dependence is seen in Figs. 5(b) and 6(b). Furthermore, in the left column of Fig. 7, a probability peak for III $\rightarrow$ I interconversion at $\Phi=90^{\circ}$ is calculated only at the collision energy which exceeds the torsional barrier $(430 \mathrm{~K})$. This peak disappears for the collision energy which is lower than the torsional barrier $(290 \mathrm{~K})$, leaving interconversion to occur only at $\Phi=0^{\circ}$ and $\Phi=180^{\circ}$. In the right column of this figure, the IV $\rightarrow$ I probabilities are again insensitive to whether the collision energy exceeds the torsional barrier.

Because III $\rightarrow$ I interconversion corresponds to rotation about the heavy $\mathrm{C}-\mathrm{C}$ bond, it is likely that it proceeds via a classical mechanism. Therefore, collision-induced III $\rightarrow$ I interconversion should occur in two regimes, determined by whether the collision energy is higher or lower than the barrier energy. In the higher-energy regime, the energy of the collision is sufficient to "knock" conformer III over the torsional barrier into conformer I. In the lower-energy regime, the collision will not be strong enough to classically surmount the barrier via purely repulsive energy transfer, so interconversion will only occur if the torsional barrier is somehow diminished by attractive interactions between the rare-gas atom and the glycine molecule.
Figure 9 illustrates the lowering effect of the colliding Ar atom on the barrier to $\mathrm{III} \rightarrow \mathrm{I}$ interconversion. The curves in the figure represent the sum of the $\mathrm{C}-\mathrm{C}$ torsional potential $v_{1}\left(\phi_{1}\right)[$ Eq. (1)] and the the glycine-Ar interaction potential $V_{I}\left(R, \phi_{1} ; \Theta, \Phi\right)$ [Eqs. (3) and (13)] for a given collision angle and various fixed values of the collision distance $R$. The solid curve in Fig. 9, which corresponds to infinite separation between the glycine molecule and the colliding atom $(R=\infty)$, is identical to that of the $\mathrm{C}-\mathrm{C}$ torsional potential presented in Fig. 4. At $R=9.2 a_{0}$, the van der Waals interaction between the Ar atom and the atoms of the glycine molecule stabilizes the entire torsional potential. At $R=8.3 a_{0}$ and $R=7.9 a_{0}$, the energy of the barrier to $\mathrm{III} \rightarrow \mathrm{I}$ interconversion continues to decrease, even as repulsive features at $\phi_{1}=180^{\circ}$ and $\phi_{1}=0^{\circ} / 360^{\circ}$ begin to emerge. Figure 9 suggests that the Ar atom substantially reduces the $\mathrm{III} \rightarrow \mathrm{I}$ interconversion barrier, thus enabling interconversion at lower collision energies. It follows that the Ar atom is a catalyst for the conformer interconversion process.

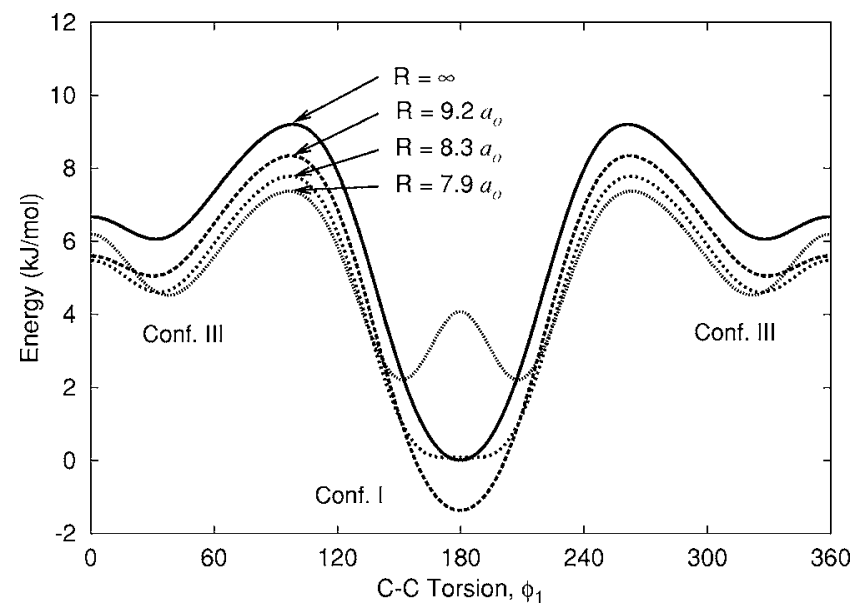

FIG. 9. The sum of the $\mathrm{C}-\mathrm{C}$ torsional potential and the glycine-Ar interaction potential at various fixed values of the collision distance $R$. The barrier to $\mathrm{III} \rightarrow \mathrm{I}$ conformer interconversion lowers as the Ar atom approaches. The collision angle used in this figure is $(\cos \Theta, \Phi)=\left(-0.3,180^{\circ}\right)$. 

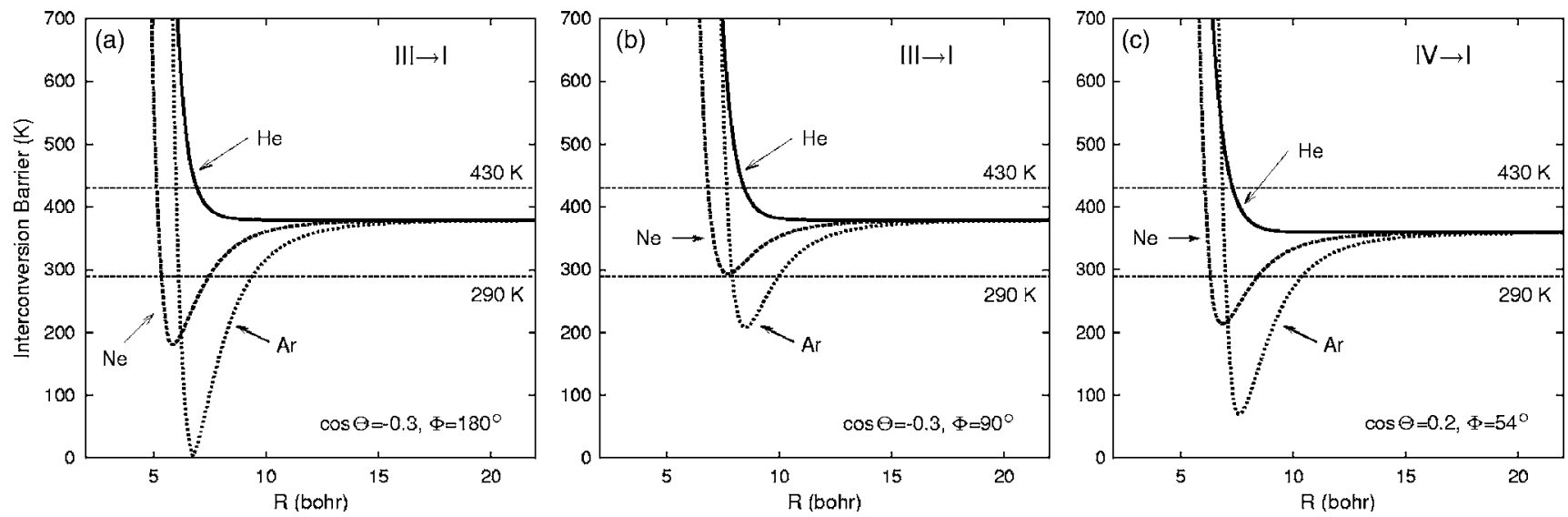

FIG. 10. [(a) and (b)] The barrier to III $\rightarrow \mathrm{I}$ interconversion as a function of the collision distance $R$. (c) The barrier to IV $\rightarrow \mathrm{I}$ interconversion as a function of the collision distance $R$. The collision angle used for each plot is specified. The $\mathrm{Ne}$ and Ar colliding atoms give rise to a substantial barrier-lowering effect; the He colliding atom does not.

In Fig. 10(a), we plot the energy of the barrier to $\mathrm{III} \rightarrow \mathrm{I}$ interconversion (relative to the minimum potential energy of conformer III at $R=\infty$ ) as a function of the collision distance $R$. The collision angle in Fig. 10(a) is the same as was considered in Fig. 9. As was seen before, the Ar atom reduces the $\mathrm{III} \rightarrow \mathrm{I}$ interconversion barrier as it approaches the glycine molecule. In fact, at $R=6.7 a_{0}$, we see that the barrier disappears entirely, suggesting that gas-phase collisions between Ar and glycine will facilitate some III $\rightarrow$ I interconversion even at very low collision energies. The $\mathrm{Ne}$ atom is seen to have a similar, but less dramatic, effect on the interconversion barrier. The He atom, which has no attractive van der Waals term in our treatment, does not lower the barrier at all.

Figure 10(a) explains the nonzero probability of $\mathrm{Ne}$ - and Ar-induced III $\rightarrow$ I interconversions at collision energies below that of the torsional barrier for the isolated glycine molecule [Figs. 5(a) and 6(a)]. A purely classical interconversion mechanism is made possible by the barrier-lowering effect of the van der Waals interaction between the Ne and Ar colliding atoms and the atoms of the glycine molecule. Figure 10(a) also explains the complete absence of He-induced $\mathrm{III} \rightarrow \mathrm{I}$ interconversion in this low-collision-energy regime [Figs. 5(a) and 6(a)]. Because the He atom does not experience attractive interactions with the atoms of the glycine molecules, it does not catalyze the interconversion process in the same way as the heavier colliding atoms.

In Fig. 10(b), we present the III $\rightarrow$ I interconversion barrier for the approach of the rare-gas atoms from a different collision angle. For the $\mathrm{Ne}$ and $\mathrm{Ar}$ atoms, the barrierlowering effect is calculated to be smaller than was seen in Fig. 10(a).

Comparison of the Ne results in Figs. 10(a) and 10(b) yields a simple explanation for the energy dependence of the interconversion probability peak at $\Phi=90^{\circ}$ in the left column of Fig. 7. The collision angle used to generate Fig. 10(a) corresponds to the III $\rightarrow$ I interconversion probability peak $\Phi=180^{\circ}$ in Fig. 7, which is found at both collision energies (290 and $430 \mathrm{~K}$ ). The collision angle used to generate Fig. 10 (b) corresponds to the probability peak $\Phi=90^{\circ}$, which is found only at the higher collision energy (430 K). In Fig. 10(a), the Ne interconversion barrier drops well below the horizontal line indicating a collision energy of $290 \mathrm{~K}$. It follows that interconversion should occur at $\Phi=180^{\circ}$, even at the lower collision energy. However, in Fig. 10(b), the $\mathrm{Ne}$ interconversion barrier does not drop below $290 \mathrm{~K}$, explaining the virtual absence of the $\Phi=90^{\circ}$ probability peak at $290 \mathrm{~K}$ in the left column of Fig. 7.

Finally in Fig. 10(c), we present the effect of the colliding atom on the IV $\rightarrow$ I interconversion barrier. The collision angle employed in this figure is $(\cos \Theta, \Phi)=\left(0.2,54^{\circ}\right)$, which corresponds to a large $\mathrm{Ne}$-induced interconversion probability at both 290 and $430 \mathrm{~K}$ in the right column of Fig. 7. As in Fig. 10(a), the Ne interconversion barrier drops below the horizontal line for $290 \mathrm{~K}$, indicating that a classical interconversion mechanism is available even at this lower collision energy. Again, the $\mathrm{He}$ atom does not give rise to any lowering of the interconversion barrier.

Given the fact that the $\mathrm{He}$ atom does not lower the IV $\rightarrow \mathrm{I}$ interconversion barrier, it is notable that substantial He-induced interconversion is calculated at collision energies below that of the interconversion barrier for the bare glycine molecule in Fig. 5(b). No classical mechanism exists for this interconversion process, since the energy of the He atom is insufficient to knock conformer IV over the barrier and the interaction potential $\mathrm{He}$ atom is unable to lower the barrier. All the interconversion probability observed in this lowenergy regime must therefore arise from quantum effects.

The distinctly quantum-mechanical nature of the IV $\rightarrow$ I interconversion process is evident from the $\phi_{3}$ vibrational energies and eigenfunctions in Fig. 4. The torsional eigenfunctions tunnel far into the interconversion barrier, so that upon collision with a rare-gas atom, the initial vibrational state of conformer IV readily couples to energetically close conformer I vibrational states. Furthermore, the large zeropoint energy of conformer IV diminishes the effective barrier height to IV $\rightarrow$ I interconversion. Both of these factors facilitate IV $\rightarrow$ I interconversion and render the calculated IV $\rightarrow$ I interconversion probabilities less sensitive to whether the collision energy is above or below the energy of the torsional barrier than the calculated III $\rightarrow$ I interconversion probabilities. 

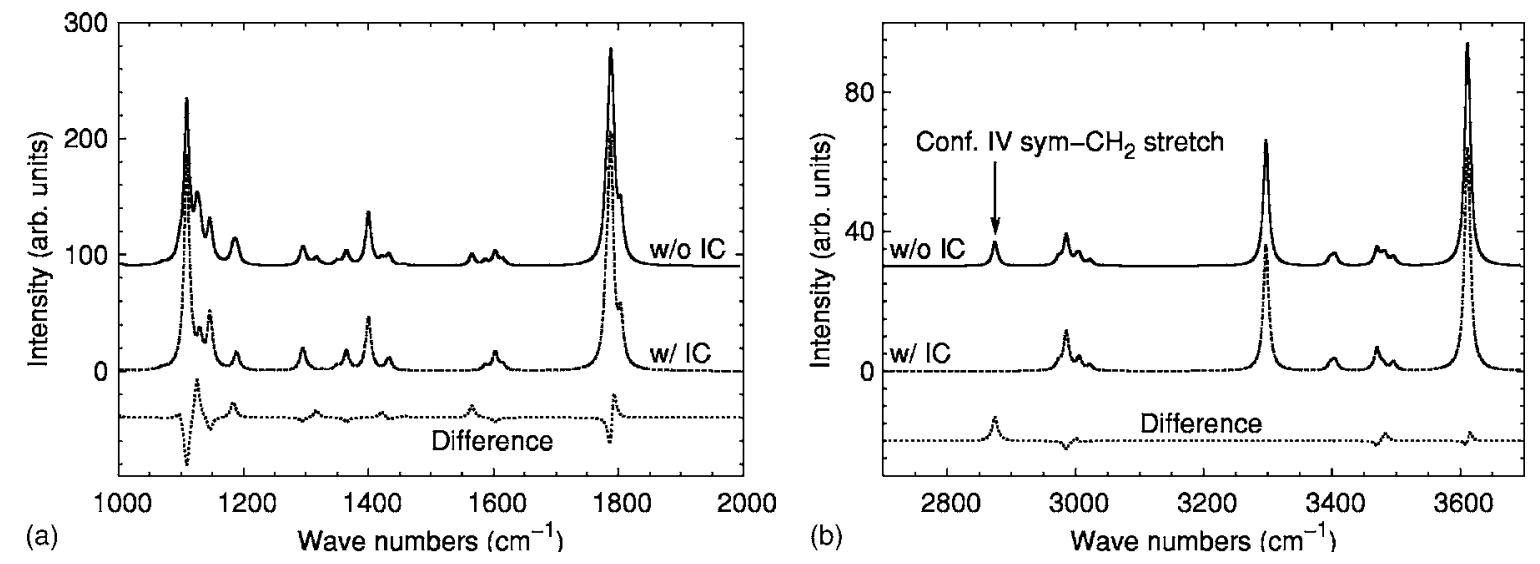

FIG. 11. Infrared-absorption spectra calculated for the distribution of glycine conformers at $438 \mathrm{~K}$ without interconversion of conformer IV (top) and with interconversion of conformer IV (middle). The bottom curve shows the difference between these two spectra. The conformer IV symmetric $-\mathrm{CH}_{2}$ stretch at $2875 \mathrm{~cm}^{-1}$ is well separated from other absorptions.

\section{Detection of conformer IV}

In the previous sections, we present evidence for the collision-induced interconversion of both conformers III and IV to conformer I. We find that the degree to which interconversion occurs is dependent on three factors: (1) the energy of collision, (2) the interaction potential between glycine and the colliding atom, and (3) the angle at which the colliding atom approaches the glycine molecule. Furthermore, based on the energy dependence of the two interconversion processes, we conclude that III $\rightarrow$ I interconversion proceeds by classical barrier-crossing mechanisms, whereas IV $\rightarrow$ I interconversion can also exhibit a quantum tunneling mechanism. However, the central question remains: Is conformer IV stable enough to be detected experimentally?

Figure 5 shows that for most of the collision energies reported in this study, we calculate very little difference in the angle-averaged probability of interconversion for the $\mathrm{III} \rightarrow \mathrm{I}$ and IV $\rightarrow$ I processes. We now consider these results in the context of two important experimental observations: (1) When the glycine molecules are evaporated at $\sim 500 \mathrm{~K}$ and subjected to free-jet expansion in a stream of argon gas, conformer III is not observed using microwave spectroscopy. ${ }^{16}$ (2) When glycine molecules are evaporated at $410-440 \mathrm{~K}$ and deposited in a matrix of $\mathrm{Ne}$, Ar, or $\mathrm{Kr}$ atoms on a sufficiently cold substrate $(<13 \mathrm{~K})$, conformer III is conclusively observed using IR spectroscopy. ${ }^{18}$

To explain observation (1), conformer III was concluded to have undergone total interconversion during free-jet expansion in the Ar carrier gas, ${ }^{17}$ which suggests that during expansion, glycine-Ar collisions occur at sufficient energy to facilitate III $\rightarrow$ I interconversion. Although it is difficult to precisely relate our single-collision calculations with the multiple-collision process that occurs experimentally, we still note that our results are consistent with this observation, predicting substantial Ar-induced III $\rightarrow$ I interconversion for collision energies at and below $500 \mathrm{~K}$ [Fig. 5(a)]. Because $\mathrm{IV} \rightarrow \mathrm{I}$ interconversion probabilities of similar magnitude are calculated in this range, it is not surprising to find that conformer IV was not observed after free- jet expansion, either.

Observation (2) has interesting consequences for our results. In the matrix-IR experiment, regardless of whether the glycine sample is cooled in the presence of $\mathrm{Ne}, \mathrm{Ar}$, or $\mathrm{Kr}$ rare-gas atoms, conformer III is still conclusively observed. This clearly indicates that substrate deposition is a more sudden cooling process (in the sense that less interconversion occurs) than free-jet expansion. In fact, because our results in Fig. 5(a) show that Ar-induced interconversion remains substantial even at $100 \mathrm{~K}$, it seems that the collisions relevant to matrix cooling occur at energies lower than those explicitly considered in the current study. As a result, without performing lower-energy scattering calculations, it is difficult to determine from our results whether conformer IV can be expected to survive under the conditions of $\mathrm{Ar}$ and $\mathrm{Ne}$ matrix cooling that preserve conformer III.

However, one important prediction can be made. Figure 5(b) shows that the total probability of He-induced IV $\rightarrow$ I conformer interconversion is virtually zero at $100 \mathrm{~K}$. Therefore, if we (a) use the conclusion from the previous paragraph that matrix deposition cooling corresponds to collision energies below $100 \mathrm{~K}$, (b) assume that cooling by deposition of molecules in He droplets is at least as sudden as matrix cooling and thus also corresponds to collisions below $100 \mathrm{~K},{ }^{64-68}$ and (c) assume that the probability for Heinduced interconversion in Fig. 5(b) remains zero for all collision energies below $100 \mathrm{~K}$, we may finally predict that conformer IV is present in experiments involving the deposition of glycine molecules in He droplets.

Huisken et al. have studied the IR spectrum of glycine molecules trapped in helium droplets and only report the detection of conformers I-III. ${ }^{21}$ However, this work focused almost exclusively on the $\mathrm{O}-\mathrm{H}$ stretching frequency of the various glycine conformers. Because the $-\mathrm{OH}$ group is not directly involved in the intramolecular hydrogen bonds of either conformer I or conformer IV, it may be very difficult to differentiate between the two conformers using that absorption band alone. In fact, a subsequent theoretical study of the anharmonic frequencies of the various glycine conformers suggests that it would be difficult to distinguish between conformers I and IV based on any of the $\mathrm{C}=\mathrm{O}, \mathrm{N}-\mathrm{H}$, or $\mathrm{O}-\mathrm{H}$ stretching absorption bands. ${ }^{24}$

We illustrate the difficulty of detecting conformer IV using IR spectroscopy in Fig. 11, which shows the calculated 
IR spectrum for two different populations of glycine. (The construction of the spectra for the figure is explained in Sec. III E.) The top spectrum in Fig. 11 [without interconversion (IC)] corresponds to the equilibrium distribution of glycine conformers I-IV at $438 \mathrm{~K}$. The calculated conformer populations at this temperature, without interconversion of conformer IV, are presented in Table I. The middle spectrum in Fig. 11 (with IC) shows the total IR spectrum of glycine at $438 \mathrm{~K}$, assuming that all of the population of conformer IV has interconverted to conformer I (see Table I, with interconversion). The bottom curve shows the difference between the total IR spectrum of glycine calculated with and without the presence of conformer IV.

Figure 11 shows how difficult it is to discern the presence of conformer IV from the combined IR spectrum of the glycine conformers. In particular, there is no unique indication of conformer IV in the $-\mathrm{OH}$ and $-\mathrm{NH}$ stretching regions above $3200 \mathrm{~cm}^{-1}$. Similarly, in the $\mathrm{C}=\mathrm{O}$ stretching region around $1800 \mathrm{~cm}^{-1}$, the small calculated differences might be difficult to characterize experimentally. However, we do find at least one clear peak that uniquely corresponds to conformer IV. It is the symmetric $-\mathrm{CH}_{2}$ stretching frequency at $2875 \mathrm{~cm}^{-1}$. We calculate no fundamental absorption band from any other conformer within $75 \mathrm{~cm}^{-1}$ of this peak, and previous anharmonic frequency calculations also suggest that it is well separated. ${ }^{24}$ Even when all overtone and combination bands are considered, the only absorption arising from a different conformer that is calculated within $20 \mathrm{~cm}^{-1}$ of this peak is a conformer II combination band calculated at $2879 \mathrm{~cm}^{-1}$. Although it is not a very strong spectral feature, our calculations suggest that the fundamental absorption band for the symmetric $\mathrm{CH}_{2}$ stretching mode at approximately $2875 \mathrm{~cm}^{-1}$ is measurable and would conclusively indicate the presence of conformer IV in a helium droplet.

\section{CONCLUSIONS}

Matrix-IR studies have successfully characterized conformers I-III of glycine, but conformer IV has yet to be conclusively observed. In light of its expected thermodynamic stability, the absence of conformer IV has previously been attributed to conformer interconversion during the dynamics of cooling.

To explore this problem, we present quantum dynamical calculations of the collision-induced interconversion of glycine conformers III and IV. For both conformers, there are cases for which we find substantial probabilities of interconversion. The calculated probabilities are dependent on several factors, including the energy of the collision, the strength of the glycine-atom interaction, and the angle at which the colliding atom approaches the glycine molecule. We conclude that III $\rightarrow$ I interconversion is a classical mechanical process that is facilitated at low collision energies by the catalytic effect of the Ne- and Ar-glycine interaction potentials. For the IV $\rightarrow$ I interconversion process, we find a similar catalytic effect as well as evidence for interconversion via quantum tunneling.

However, our calculations present little indication that conformer IV interconverts more readily than the consis- tently observed conformer III. Although we cannot conclusively address whether IV $\rightarrow$ I interconversion occurs during $\mathrm{Ne}$ - and Ar-matrix depositions, our evidence suggests that conformer IV will be found in helium droplets. Anharmonic vibrational frequency calculations suggest that the redshifted IR-absorption band for the symmetric $-\mathrm{CH}_{2}$ stretching mode will provide a clear indication of the elusive conformer.

The results and conclusions presented in this work are, as always, subject to the quality of the assumptions employed. In particular, the calculated interconversion probabilities are certainly dependent on the torsional potentials and the glycine-atom interaction potentials that we have used. Furthermore, the employed dynamical approximations could possibly affect the details of our calculations. Throughout the paper, we have stressed the limitations of our singlecollision treatment of collisional cooling. We also note that steering effects, non- $S$-wave scattering transitions, and the explicit participation of nontorsional modes during interconversion have been neglected. However, regardless of these approximations, the current paper presents a detailed theoretical treatment of collision-induced conformer interconversion that will form the basis of our future efforts to accurately describe dynamical cooling.

\section{ACKNOWLEDGMENTS}

Two of the authors (T.F.M. and D.C.C.) acknowledge fellowships from the National Science Foundation and the Leverhulme Trust, respectively. Another author (A.J.H.M.M.) acknowledges funding from the University of Sheffield. The authors thank Dr. Pierre Çarçabal for his helpful comments.

${ }^{1}$ G. Junk and H. Svec, J. Am. Chem. Soc. 85, 839 (1963).

${ }^{2}$ K. Iijima, K. Tanaka, and S. Onuma, J. Mol. Struct. 246, 257 (1991).

${ }^{3}$ S. Vishveshwara and J. A. Pople, J. Am. Chem. Soc. 99, 2422 (1977).

${ }^{4}$ H. L. Sellers and L. Schäfer, J. Am. Chem. Soc. 100, 7728 (1978).

${ }^{5}$ R. D. Brown, P. D. Godfrey, J. W. V. Storey, and M. P. Bassez, J. Chem. Soc., Chem. Commun. 1978, 547.

${ }^{6}$ R. D. Suenram and F. J. Lovas, J. Mol. Spectrosc. 72, 372 (1978).

${ }^{7}$ L. Schäfer, H. L. Sellers, F. J. Lovas, and R. D. Suenram, J. Am. Chem. Soc. 102, 6566 (1980).

${ }^{8}$ R. D. Suenram and F. J. Lovas, J. Am. Chem. Soc. 102, 7810 (1980).

${ }^{9}$ M. Ramek, V. K. W. Cheng, R. F. Frey, S. Q. Newton, and L. Schäfer, J. Mol. Struct.: THEOCHEM 235, 1 (1991).

${ }^{10}$ R. F. Frey, J. Coffin, S. Q. Newton, M. Ramek, V. K. W. Cheng, F. A. Momany, and L. Schäfer, J. Am. Chem. Soc. 114, 5369 (1992).

${ }^{11}$ J. H. Jensen and M. S. Gordon, J. Am. Chem. Soc. 113, 7917 (1991).

${ }^{12}$ A. G. Császár, J. Am. Chem. Soc. 114, 9568 (1992).

${ }^{13}$ C. H. Hu, M. Shen, and H. F. Schaefer III, J. Am. Chem. Soc. 115, 2923 (1993).

${ }^{14}$ F. Barone, C. Adamo, and F. Lelj, J. Chem. Phys. 102, 364 (1995).

${ }^{15}$ A. G. Császár, J. Mol. Struct. 346, 141 (1995).

${ }^{16}$ P. D. Godfrey and R. D. Brown, J. Am. Chem. Soc. 117, 2019 (1995).

${ }^{17}$ P. D. Godfrey, R. D. Brown, and F. M. Rodgers, J. Mol. Struct. 376, 65 (1996).

${ }^{18}$ I. D. Reva, A. M. Plokhotnichenko, S. G. Stepanian, A. Y. Ivanov, E. D. Radchenko, G. G. Sheina, and Y. P. Blagoi, Chem. Phys. Lett. 232, 141 (1995).

${ }^{19}$ A. Y. Ivanov, A. M. Plokhotnichenko, V. Izvekov, G. G. Sheina, and Y. P. Blagoi, J. Mol. Struct. 408/409, 459 (1997).

${ }^{20}$ S. G. Stepanian, I. D. Reva, E. D. Radchenko, M. T. S. Rosado, M. L. T. S. Duarte, R. Fausto, and L. Adamowicz, J. Phys. Chem. A 102, 1041 (1998).

${ }^{21}$ F. Huisken, O. Werhahn, A. Y. Ivanov, and S. A. Krasnokutski, J. Chem. Phys. 111, 2978 (1999). 
${ }^{22}$ A. Y. Ivanov, G. G. Sheina, and Y. P. Blagoi, Spectrochim. Acta, Part A 55, 219 (1999).

${ }^{23}$ R. Ramaekers, J. Pajak, B. Lambie, and G. Maes, J. Chem. Phys. 120, 4182 (2004).

${ }^{24}$ O. Bludský, J. Chocholoušová, J. Vacek, F. Huisken, and P. Hobza, J. Chem. Phys. 113, 4629 (2000).

${ }^{25}$ G. M. Chaban, J. O. Jung, and R. B. Gerber, J. Phys. Chem. A 104, 10035 (2000).

${ }^{26}$ T. F. Miller III and D. C. Clary, Phys. Chem. Chem. Phys. 6, 2563 (2004).

${ }^{27}$ B. Brauer, G. M. Chaban, and R. B. Gerber, Phys. Chem. Chem. Phys. 6, 2453 (2004)

${ }^{28}$ H. Q. Ai, Y. X. Bu, and Z. D. Chen, J. Chem. Phys. 118, 1761 (2003).

${ }^{29}$ Y. J. Kwan, S. B. Charnley, H. C. Huang, W. L. Tseng, and Z. Kisiel, Astrophys. J. 593, 848 (2003).

${ }^{30}$ T. F. Miller III and D. C. Clary, J. Chem. Phys. 116, 8262 (2002).

${ }^{31}$ T. F. Miller III and D. C. Clary, J. Chem. Phys. 119, 68 (2003).

${ }^{32}$ R. S. Ruoff, T. D. Klots, T. Emilsson, and H. S. Gutowsky, J. Chem. Phys. 93, 3142 (1990).

${ }^{33}$ P. Butz, R. T. Kroemer, N. A. Macleod, and J. P. Simons, Phys. Chem. Chem. Phys. 4, 3566 (2002).

${ }^{34}$ P. D. Godfrey and R. D. Brown, J. Am. Chem. Soc. 120, 10724 (1998).

${ }^{35}$ G. M. Florio, R. A. Christie, K. D. Jordan, and T. S. Zwier, J. Am. Chem. Soc. 124, 10236 (2002).

${ }^{36}$ A. Bastida, J. Zúñiga, A. Requena, B. Miguel, J. A. Beswick, J. Viguẽ, and N. Halberstadt, J. Chem. Phys. 116, 1944 (2002).

${ }^{37}$ D. Miller, in Atomic and Molecular Beam Methods, edited by G. Scoles (Oxford University Press, Oxford, 1988), pp. 14-53.

${ }^{38}$ R. Levine and R. Bernstein, Molecular Reaction and Chemical Reactivity (Oxford University Press, Oxford, 1987).

${ }^{39}$ D. W. Zhang, M. L. Wang, and J. Z. H. Zhang, J. Phys. Chem. A 107, 7106 (2003).

${ }^{40}$ B. L. Lan and J. M. Bowman, J. Chem. Phys. 101, 8564 (1994).

${ }^{41}$ K. M. Christoffel and J. M. Bowman, J. Chem. Phys. 112, 4496 (2000).

${ }^{42}$ D. C. Clary and A. J. H. M. Meijer, J. Chem. Phys. 116, 9829 (2002).

${ }^{43}$ C. Schwartz, J. Math. Phys. 26, 411 (1985).

${ }^{44}$ D. T. Colbert and W. H. Miller, J. Chem. Phys. 96, 1982 (1992).
${ }^{45}$ G. C. Groenenboom and D. T. Colbert, J. Chem. Phys. 99, 9681 (1993).

${ }^{46} \mathrm{G}$. C. Groenenboom (private communication).

${ }^{47}$ Note the error with the signs in Ref. 45 (see Ref. 46).

${ }^{48}$ D. C. Clary and G. J. Kroes, in Advances in Chemical Kinetics and Dynamics (Wiley, New York, 1995), Vol. 2A, pp. 135-168.

${ }^{49}$ D. C. Clary, Mol. Phys. 51, 1299 (1984).

${ }^{50}$ J. M. Hutson, D. C. Clary, and J. A. Beswick, J. Chem. Phys. 81, 4474 (1984).

${ }^{51}$ R. A. Aziz and M. J. Slaman, Chem. Phys. 130, 187 (1989).

${ }^{52}$ J.-U. Grabow, A. S. Pine, G. T. Fraser, F. J. Lovas, R. D. Suenram, T. Emilsson, E. Arunan, and H. S. Gutowsky, J. Chem. Phys. 102, 1181 (1995).

${ }^{53}$ S. K. Gray and D. E. Manolopoulos, J. Chem. Phys. 104, 7099 (1996).

${ }^{54}$ S. K. Gray and C. E. Wozny, J. Chem. Phys. 91, 7671 (1989).

${ }^{55}$ A. J. H. M. Meijer and E. M. Goldfield, J. Chem. Phys. 108, 5404 (1998).

${ }^{56}$ See also http://www.cs.utexas.edu/users/flame/goto/.

${ }^{57}$ M. Frigo and S. G. Johnson, in Proceedings of the 1998 IEEE International Conference on Acoustics Speech and Signal Processing (IEEE, New York, 1998), Vol. 3, pp. 1381-1384.

${ }^{58}$ M. Frigo and S. G. Johnson, special issue on Program Generation, Optimization, and Adaptation [Proc. IEEE 93, 216 (2005)].

${ }^{59}$ See also http://www.fftw.org.

${ }^{60}$ D. H. Zhang and J. Z. H. Zhang, J. Chem. Phys. 101, 1146 (1994).

${ }^{61}$ P. Pernot and W. A. Lester, Int. J. Quantum Chem. 40, 577 (1991).

${ }^{62}$ P. Pernot, R. M. Grimes, W. A. Lester, and C. Cerjan, Chem. Phys. Lett. 163, 297 (1989).

${ }^{63}$ M. L. Frisch et al., GAussian03, Revision B.05, Gaussian, Inc, Wallingford, CT, 2004.

${ }^{64}$ N. Pörtner, A. F. Vilesov, and M. Havenith, Chem. Phys. Lett. 343, 281 (2001).

${ }^{65}$ C. Callegari, K. K. Lehmann, R. Schmied, and G. Scoles, J. Chem. Phys. 115, 10090 (2001).

${ }^{66}$ K. Nauta and R. E. Miller, Science 283, 1895 (1999).

${ }^{67}$ K. Nauta and R. E. Miller, Science 287, 293 (2000).

${ }^{68}$ J. P. Toennies and A. F. Vilesov, Annu. Rev. Phys. Chem. 49, 1 (1998). 